\title{
Towards Human Energy Expenditure Estimation Using Smart Phone Inertial Sensors
}

\author{
Božidara Cvetković ${ }^{1,3}$, Boštjan Kaluža ${ }^{1,3}$, Radoje Milić ${ }^{2}$, and Mitja Luštrek ${ }^{1,3}$ \\ 1 Jožef Stefan Institute, Department of Intelligent Systems, \\ Jamova ceta 39, 1000 Ljubljana, Slovenia \\ \{boza.cvetkovic, bostjan.kaluza, mitja.lustrek\}@ijs.si \\ http://ijs.dis.si \\ 2 University of Ljubljana, Faculty of Sports \\ Gortanova 22, Ljubljana, Slovenia \\ radoje.milic@fsp.uni-lj.si \\ http://www.fsp.uni-lj.si/ \\ 3 Jožef Stefan International Postgradute Shool \\ Jamova ceta 39, 1000 Ljubljana, Slovenia \\ http://www.mps.si
}

\begin{abstract}
This paper is focused on a machine-learning approach for estimating human energy expenditure during sport and normal daily activities. The paper presents technical feasibility assessment that analyses requirements and applicability of smart phone sensors to human energy expenditure. The paper compares and evaluates three different sensor configuration sets: (i) a heart rate monitor and two standard inertial sensors attached to the users thigh and chest; (ii) a heart rate monitor with an embedded inertial sensor and a smart phone carried in the pocket; and (iii) only a smart phone carried in the pocket. The accuracy of the models is validated against indirect calorimetry using the Cosmed system and compared to a commercial device for energy expenditure SenseWear armband. The results show that models trained using relevant features can perform comparable or even better than available commercial device.
\end{abstract}

Keywords: human energy expenditure, physical activity, wearable sensors, embedded smart phone sensors, regression

\section{Introduction}

Medical research has shown that a sufficient amount of physical activity has a positive impact on health and well-being regardless of the age [1-3], and that physical inactivity is one of the leading causes of death worldwide [4]. Although this is widely accepted as a fact, only a small fraction of the population is engaged in regular or sufficient exercise. The key reasons usually include lack of time and fast pace of life. This means that a persuasive technology that would encourage exercise could be greatly beneficial to many people. An important component of such a technology is a possibility to quantify the amount of physical activity 
performed during the day. Furthermore, the amount of physical activity can be used to manage the diet of both, a healthy individual or someone who suffers from dietary disease (e.g., diabetes). This raises the question of how can we unobtrusively measure the amount of physical activity.

The cost of physical activity is usually expressed in metabolic equivalents of task (MET), where 1 MET is defined as the energy expended at rest. MET values range from 0.9 (sleeping) to over 20 in extreme exertion. Various methods can be used to reliably estimate energy expenditure (EE). Direct calorimetry [5] measures the heat produced by human body while exercising. This is the most accurate method, but it can be used only in a controlled environment such as a laboratory. Indirect calorimetry [6] measures the carbon dioxide production and oxygen consumption during rest and steady-state exercise. This method can be used outside the laboratory, but it cannot be used in everyday life since its usage requires a breathing mask. Doubly labelled water [7] is a gold standard: it measures the amount of exhaled carbon dioxide by tracking its amount in water which is labelled by deuterium and oxygen-18. This method can be used in everyday life, but it measures the energy expended over longer periods of time and is rather expensive. Finally, the most affordable approach is a commercial wearable sensors such as inertial sensors embedded in armbands and other devices, which are moderately accurate and reliable. These can be used in everyday life and the EE estimation can be done over shorter periods of time.

Inertial sensors as motion sensors are already very popular in different domains such as gaming industry $[8,9]$, healthcare and medicine [10-12] and security [13]. Their accessibility, ease of use, and understandable concept of accelerometry help broadening its applicability domains on a daily basis. For instance, even running shoes can contain an accelerometer, while an average smart phone contains a wide range of sensors, including an accelerometer. The fact that many of us hardly leave our home without a smart phone made an important impact on development, realization and acceptance of novel smart applications.

This paper thus studies EE estimation using inertial sensors that are present in every commercial armband for EE estimation. It presents a machine-learning approach, and is concerned both with normal daily activities as well as exercise. We compare three sensor configuration sets: (i) a chest strap heart rate monitor and two standard inertial sensors attached at the thigh and chest; (ii) a chest strap heart rate monitor with an embedded inertial sensor and a smart phone carried in the pocket; and (iii) a smart phone carried in the pocket. The accuracy of the EE estimation models is validated against indirect calorimetry approach using the Cosmed system [14] and a commercial device for EE, the SenseWear armband [15]. SenseWear armband show to have the lowest error in free-living situations [16] among the popular physical activity monitors.

The goal of the paper is two-fold: (i) to compare three sensor sets from the one requiring the most dedicated devices, to the one requiring only what most people carry around, i.e., smartphone; and (ii) to present the methodology for the development of an accurate machine-learning model for the EE estimation that can be used on a variety of devices. 
The rest of the paper is structured as follows. Section 2 presents the related work; Section 3 presents the sensors used for data collection presented in Section 4. Section 5 contains the details on data pre-processing and feature selection, while section 6 shows experimental results. Section 7 concludes the paper.

\section{Related Work}

There is a growing trend in development of smart-phone applications for health monitoring, fitness trainers and EE estimation, which is evident at the application markets for mobile platforms. These applications can be divided into two categories; those that use the accelerometer embedded in the smart phone to estimate EE based on the number of steps the user does over one day [17] (essentially pedometers); and those that estimate the intensity of the performed activity and thus the expended energy directly, for example, MyFitnessCompanion [18]. Pedometers can be used only to detect the ambulatory activities, such as walking or running, and not their intensities. MyFitnessCompanion application can detect the intensity, but it has one major shortfall: the user must manually define which activity is being performed. The EE estimation is afterward based on a predefined energy estimation values taken from the Compendium of Physical Activities [19].

Most of the methods based on machine-learning techniques estimate EE using wearable smart phone sensors and seek linear or nonlinear relations between the energy expenditure and the accelerometer outputs. The most basic methods use an accelerometer and a linear regression model. The estimation accuracy can be improved by multiple regression models [20] and complex features [21]. A regression method by Crouter et al. [22], which is currently among the most accurate approaches, uses an accelerometer attached to the hip. In the first step, it classifies a person's activity into sitting, ambulatory activity or lifestyle activity. In the second step, it uses a linear regression model for the ambulatory activity and an exponential regression model for the lifestyle activity. Sitting is always considered to have the energy expenditure of 1 metabolic equivalent of task (MET, 1 MET is the energy expended at rest). A drawback of this method is the exclusion of some important activities, such as cycling, and a larger EE estimation error contributed by the upper body, which is caused by sensor placed at the hip.

There are many available commercial devices, dedicated to EE estimation such as Actigraph [23] and Nike+ [24]. One of the most accurate is the SenseWear armband $[15,16]$ device, comprising a variety of sensors such as skin temperature detection, heat flux, galvanic skin response and accelerometer. EE estimation is based on activity predicted from the armband accelerometer and data analysis from other sensors. It is highly accurate in EE estimation during sports activities; however, it performs with lower accuracy in case of normal daily activities such as chores.

This paper present an approach with an additional regression model, which is based on the current user's activity in case when at least two sensors are used. 
The activity is automatically recognised using an activity-recognition classifier. The approach is demonstrated on three sensor configuration sets, including a single smart phone. In this case the activity is not used as one of the features.

The experimental results compare the proposed approach against SenseWear armband developed by Bodymedia [15], and the indirect calorimeter measurement performed by portable Cosmed $k 4 b^{2}$ [14].

\section{Sensors}

We considered five different sensors as shown in Figure 1: first, a wireless Shimmer inertial sensor [25] (Figure 1a); second, an inertial sensor integrated in the Zephyr bioharness chest strap citezephyr (Figure 1b), which also measured heart rate; third, an inertial sensor embedded in a smart phone, in our case a Samsung Galaxy SII smart phone [27] (Figure 1c). Fourth, reference energy expenditure values, which are used to develop and evaluate EE estimation models, were measured using a portable indirect calorimetery system Cosmed $k 4 b^{2}$ [14] (Figure 1d), which is considered a gold standard for EE estimation. And fifth, in addition to reference energy expenditure values, we used a commercial product SenseWear armband developed by Bodymedia [15] (Figure 1e) as another baseline for result comparison.

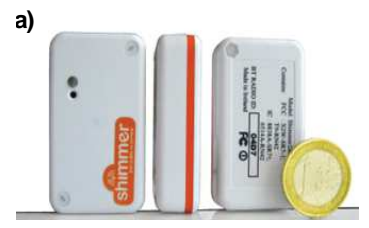

c)

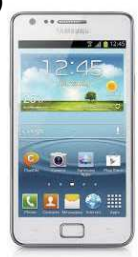

b)
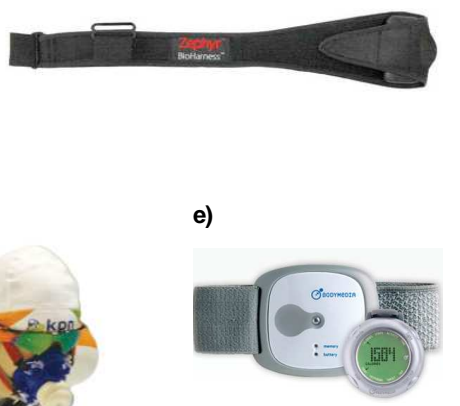

Fig. 1. Used sensors: a) a tri-axial Shimmer inertial sensor, b) a Zephyr bioharness chest-strap with tri-axial inertial sensor and hart rate monitor, c) a Samsung Galaxy SII smart phone, d) a gold standard portable indirect calorimetery Cosmed $k 4 b^{2}$ device and e) a SenseWear device for EE estimation.

To study the feasibility of EE estimation using smart phone, we approached by gradually decreasing the number of used sensors and evaluating the accuracy of the machine-learning approach for EE estimation (explained in Section 6). Particularly, we used three sensor configuration sets as shown in Figure 2. The 
full set consists of two tri-axial inertial sensors, one attached at the chest and the other at the thigh, and a heart rate monitor (Figure 2a). We assume that the full set should provide the lowest EE estimation error.

In the second, embedded set (Figure 2b), we replaced the dedicated inertial sensors with an embedded inertial sensors as follows: the inertial sensor on the chest is replaced with the inertial sensor embedded in the Zephyr chest strap, while the inertial sensor attached to the thigh is replaced with a smart phone containing an embedded inertial sensor. The embedded set is expected to perform the same or worse than the first one, since embedded sensors may contribute some measurement noise.

In the last sensor configuration, the smart-phone set, we removed the chest strap, and used a smart phone embedded inertial sensor only (Figure 2c). The smart-phone set is expected to achieve worse performance than the second configuration. We are interested if the EE estimation error is still in the range of acceptable values.

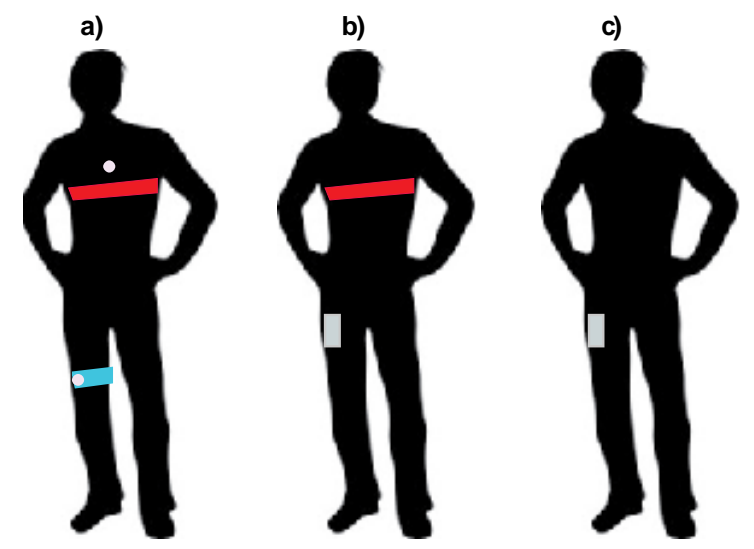

Fig. 2. Three sensor configuration sets: a) the full set, a tri-axial Shimmer inertial sensor attached to chest and thigh and a Zephyr bioharness chest strap, b) the embedded set, a Zephyr bioharness chest strap and smart phone, and c) the smart-phone set, only smart phone.

\section{Data Collection}

A machine-learning approach requires a high quality dataset. For the purpose of EE estimation we must primarily have data with accurate EE values while performing different activities and secondly measurements of various sensors at the same time.

The dataset was collected in a controlled laboratory environment at the University of Ljubljana, Faculty of Sports. The laboratory was equipped with fitness 
equipment, such as treadmill and indoor bicycle, and a portable Cosmed $k 4 b^{2}$ system, which was used to measure reference EE values. The person being measured was supplied with all the sensors mentioned in Section 3; two Shimmer inertial sensors, attached at the chest and the thigh, a Zephyr bioharness, a SenseWear armband, a smart phone, carried in the right pocket downwards with screen towards the body, and the Cosmed system.

The person performed predefined scenarios presented in Table 4, which aim to capture the intensity and EE during free-living activities.

Table 1. Scenarios and activities performed by a person. The last column represents the average measured MET by Cosmed system.

\begin{tabular}{llc}
\hline Scenario & Atomic activities & Average MET \\
\hline Lying & lying & 1.2 \\
\hline \multirow{2}{*}{ Basic postures } & sitting & 1.15 \\
& standing & 1.21 \\
& walking & 1.37 \\
& transition & 1.97 \\
\hline \multirow{2}{*}{ Additional postures } & allfours & 2.22 \\
& kneeling & 1.45 \\
& sitting & 2.39 \\
Ofiice activities & standing & 2.21 \\
Lying excersising & leaning & 1.85 \\
\hline \multirow{2}{*}{ Light chores } & walking & 2.75 \\
\hline \multirow{2}{*}{ Scrubbing the floor } & sitting & 1.17 \\
\hline \multirow{2}{*}{ Shoveling } & lying & 2.12 \\
\hline \multirow{2}{*}{ Walking } & standing & 1.68 \\
\hline \multirow{2}{*}{ Stationary cycling } & walking & 2.02 \\
\hline \multirow{2}{*}{ Running } & kneeling & 3.20 \\
\hline
\end{tabular}

In more detail, the normal day scenarios are interpreted as follows:

- Lying scenario corresponds to sleeping period when the person's metabolic rate slows down.

- Basic postures scenario corresponds to a normal lazy day. 
- Additional postures scenario corresponds to a dynamic day (playing with children on the floor).

- Office activities scenario corresponds to time at the office.

- Lying exercising scenario corresponds to stretching on the floor.

- Light chores scenario corresponds to cooking, serving food, washing dishes, folding/hanging laundry, shopping.

- Scrubbing the floor scenario corresponds to cleaning the floor on hands and knees.

- Shovelling scenario corresponds to digging and shovelling snow.

The sports activities, such as walking, running and cycling, were performed on a treadmill and a stationary bicycle under controlled speed. The EE in a specific scenario differs among activities, for example, standing in the basic posture scenario has EE of 1.21 MET, while standing in the shovelling scenario has EE of 3.06 MET. This indicates that if we can accurately (i) recognise the activity and (ii) detect the intensity, then we can reliably estimate EE, hence EE estimation error should decrease.

We recruited ten healthy people, eight males and two females with different fitness capabilities, to perform the scenarios presented in Table 4 . Their body mass index (BMI) ranged from 20 to 28.9. Each person performed all the scenario activities while equipped with the sensors shown in Figure 1; only a single person did not carry the smart phone due to technical issues. The reference EE measured by Cosmed ranged from 0.9 MET to 12 MET.

\section{The Methodology}

\subsection{Data Pre-processing}

The collected data can be divided into two groups according to the modality. The first group corresponds to signals from inertial sensors; even-though it is collected from three devices (Shimmer, Zephyr, smart phone), it should be processed using the same mechanism. The second group corresponds to signals from heart rate sensor; this data is processed only if the sensor set configuration uses heart rate sensor).

The stream of data is collected from the connected devices and split into 10 seconds windows, each window overlapping with the previous one by one half of its length. For each overlapping window a set of features is computed. A single inertial sensor contributes 66 features, a heart rate monitor contributes one feature, and the recognised activity (by additional classifier) is considered as an additional feature. The reader is referred to $[28,29]$ for details on the activity recognition methods and adaptation of activity recognition. The heart rate feature is computed as an average heart rate in the time window.

Features computed from inertial sensor are partially adopted from Tapia [30] (43 features) and partially developed by us ( 25 features). Adopted features are: mean of absolute signal value, cumulative sum over absolute signal value, entropy, quartiles, variance, inter quartile range, Fourier transform features and 
mean crossing rate. Features developed by us are: signal peak count, cumulative sum over peak absolute value, cumulative sum over signal absolute value, cumulative sum over signal absolute value after band-pass filtering, cumulative square sum over signal absolute value after band-pass filtering, cumulative sum of square components, square of cumulative sum of components after band-pass filtering, velocity, kinetic energy, vector length, integration of area under vector length curve. In total, there are 136 features for full set and embedded set and 67 features for smart-phone set. These features form a feature vector, which if fed into a machine-learning algorithm to train a model.

\subsection{Feature Selection}

To filter out redundant features and to reduce calculation complexity we performed feature selection using ReliefF method [31], which returns the features ranked by its predictive power; the returned rank represents the importance of the feature. We proceeded with the first half of the features with a positive rank value, which were then used in the feature vector. The feature selection was performed for each sensor set configuration separately. Note, that feature selection in embedded set and smart phone set was performed with nine people only, since the smart phone data was missing for one person.

Full set: the first five highest ranked features derived from inertial sensors are velocity (2 features), cumulative sum over peak absolute value (1), prevalent activity ( 1 feature), cumulative sum over signal absolute value (1 feature). The highest ranked feature is as expected the heart rate. The result is 68 highest ranked features, one from which is heart rate and one is prevalent activity. The prevalent activity can have one of ten values: lying, sitting, standing, standing leaning, allfours, kneeling, transition, walking, running or cycling.

Embedded set: seven features were ranked negative and were discarded immediately leaving 129 features for selection. The cut of point left 64 features. Highest ranked features are quartiles ( 3 features) and peak count of thigh (1 feature) and prevalent activity. The prevalent activity can have one of seven values: lying, upright position, allfours, transition, walking, running or cycling. Heart rate feature is at seventh place.

Smart-phone set: three features were ranked negative and were discarded immediately leaving 64 for the regression model. Since in this configuration we use only one sensor, none of the remaining features are discarded to have a comparable number of features to previous configurations. Highest ranked features are quartiles ( 4 features) and peak count ( 1 feature). This set does not contain feature of prevalent activity.

It is interesting to note that our developed features turned out to have better predictive power that the features developed by Tapia [30] in full set. Also at least one of our features were ranked in the first five in the embedded and smartphone set.

Table 2 shows the number of features per sensor before and after feature selection for all the configuration sets. 
Table 2. Number of features per sensor before and after feature selection.

\begin{tabular}{lcccccc}
\hline \multirow{2}{*}{ Sensors } & \multicolumn{2}{c}{ Full set } & \multicolumn{5}{c}{ Embedded set Smart-phone set } \\
\cline { 2 - 7 } & Before & After & Before & After & Before & After \\
\hline Shimmer chest & 67 & 39 & - & - & - & - \\
Shimmer thigh & 67 & 27 & - & - & - & - \\
Zephyr inertial sensor & - & - & 67 & 37 & - & - \\
Zaphyr heart rate & 1 & 1 & 1 & 1 & - & - \\
Smart phone inertial sensor & - & - & 67 & 25 & 67 & 64 \\
Activity* & 1 & 1 & 1 & 1 & - & - \\
\hline Total & 136 & 68 & 136 & 64 & 67 & 64 \\
\hline
\end{tabular}

\subsection{The Approach}

The main goals are: first, to build an effective model that can be used in each of the sensor configurations; second, to measure EE error; and third, to evaluate the feasibility of EE estimation using a smart phone.

The machine-learning approach, which is shown on Figure 3, comprises four steps. The first step is feature selection, while the second step selects the best performing algorithm. We compared the following six regression algorithms implemented in the Weka suite [32]: support vector regression (SVR), linear regression (LR), multilayer perceptron (MLP), M5-Rules (M5Rules), regression tree M5P (M5P) and regression tree REPTree (REPTree). The third step evaluates the models and analyses the distribution of mean absolute error. In case the error analysis shows that the model could be enhanced, the fourth step reconstructs and updates the regression model based on observation from previous step. When enhancement is not possible, we assume this is the final model. The experiment section reports on each configuration separately.

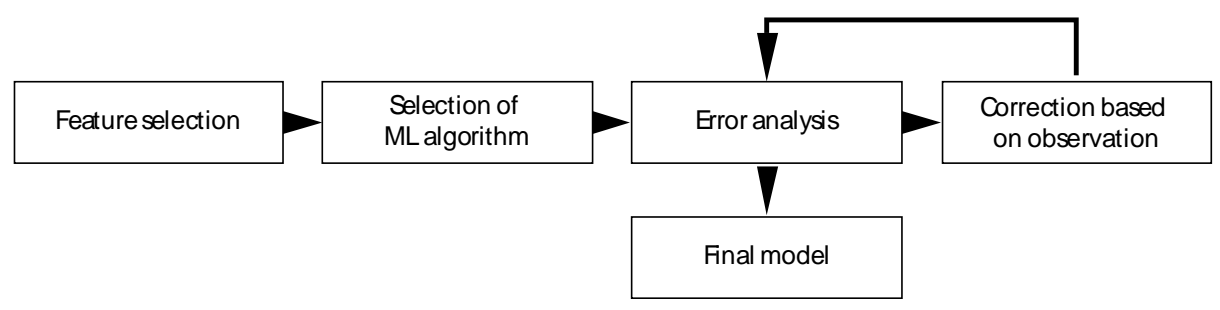

Fig. 3. The workflow of the development of the machine-learning approach.

\section{Experimental Results}

For the experiment we collected the data described in Section 4 and processed as explained in Section 5. 
The models are evaluated with two types of error measures. The first error measure is the mean absolute error (MAE) shown in Equation 1. It measures the absolute difference between the predicted and true value. The second error measure is the mean absolute percentage error (MAPE) shown in Equation 2. In contrast to MAE, it measures percentage of the error between the predicted and true value. We believe this error measure might be misleading, since errors are higher when the true value is low and lower when the true value is high; however, we report this measure to ensure comparability with other papers reporting it.

$$
\begin{gathered}
M A E=\frac{1}{n} \sum_{1}^{n}\left|M E T_{\text {true }}-M E T_{\text {predicted }}\right| . \\
M A P E=\frac{1}{n} \sum_{1}^{n}\left|\frac{M E T_{\text {true }}-M E T_{\text {predicted }}}{M E T_{\text {true }}}\right| .
\end{gathered}
$$

\subsection{The Full Set}

The full-set configuration dataset is described with the first 68 features ranked by the feature selection procedure described in Section 5.2. The performance comparison of different machine-learning algorithms is summarised in Table 4. Each column corresponds to MAE errors of a selected machine-learning approach, while each row corresponds to particular sensor set configuration. The results of the compared algorithms are obtained using 10-fold cross-validation with leave-one-person-out-approach. For example, the first row in Table 4, that is, full configuration, shows that the lowest MAE of 0.65 MET is achieved by support vector regression (SVR). Furthermore, Figure 4 shows the EE estimation error for the support vector regression model. The horizontal axis represents the true MET values, that is, the reference values obtained with Cosmed device, while vertical axis represents the estimated MET values, obtained with SVR model. We can observe that the predicted value is close to the true value. The highest error occurs in case of activities with EE from 2 MET to 8 MET. The highest error occurs in case of activities with the lowest intensity and the highest intensity.

This model alone is already more accurate than SensWear; however, the error analysis shows that the major error occurs in case of ambulatory activities such as walking, running, cycling and activity standing, thus can be improved. In our previous research [33], we observed that training per-class regression model for running and cycling contributes to lower EE estimation error. An improvement upon the error analysis includes training separate regression models for three activities, that is, running, walking and standing. This approach gives the final result, which is shown in Table 4. The MAE is decreased by $0.05 \mathrm{MET}$ and the MAPE is decreased by 3 percentage points. The main insight from the error analysis is that it is beneficial to train separate regression model for individual activities. 


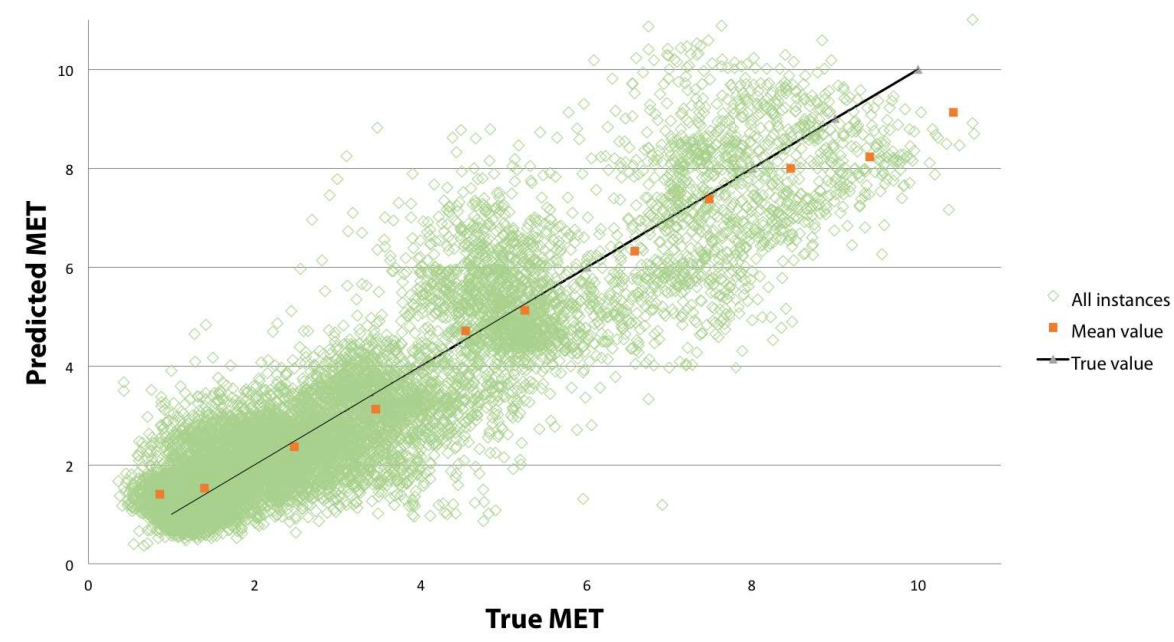

Fig. 4. Predicted MET vs. true MET for full-set configuration.

\subsection{Embedded Set}

The embedded-set configuration dataset is described with the first 64 features ranked by the feature selection procedure. The results of the compared algorithms are obtained using 9-fold cross-validation with leave-one-person-outapproach and are shown in the second row in Table 3. The lowest MAE is achieved using SVR. The SVR model outperforms the SensWear; however, the error analysis shows that running activity, if merged into a single regression model, can contribute to lower error.

A retraining of the independent model decreased MAE by 8 percentage point. The MAPE of the final model is higher, but still comparable to SenseWear's.

Figure 5 shows the EE estimation error for the embedded set model trained with SVR. It shows that the error is lower for activities with EE from 2 MET to $7 \mathrm{MET}$, while the error is higher for activities with the lowest intensity and the highest intensity.

\subsection{Smart-phone Set}

The smart-phone set configuration dataset is described with the first 64 features ranked by the feature selection procedure. The results of the compared algorithms are obtained using 9-fold cross-validation with leave-one-person-outapproach, which is shown in the third row in Table 3. The SVR outperformed other algorithms by training a model with MAE of 0.83 MET. The model outperforms Senswear's by 3 percentage points according to MAE, while MAPE of the final model is higher, but still comparable to SenseWeare's.

The error pattern is similar as in the previous configurations; however, in this case we cannot perform the adaptation procedure due to unknown activity 


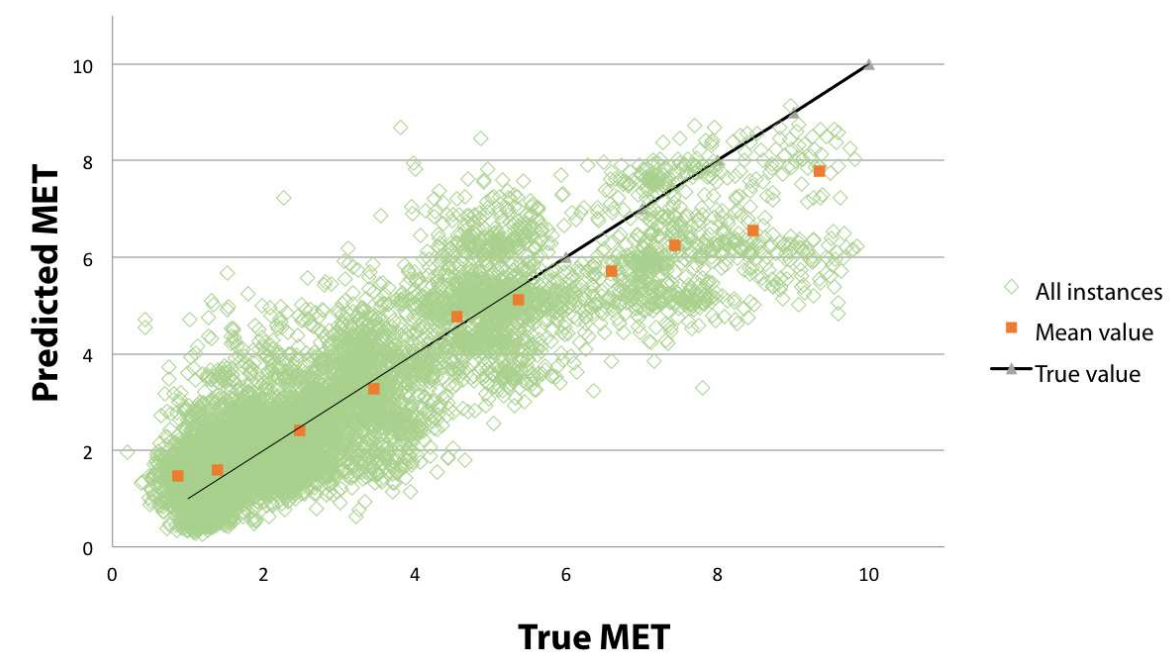

Fig. 5. Predicted MET vs. true MET for embedded-set configuration.

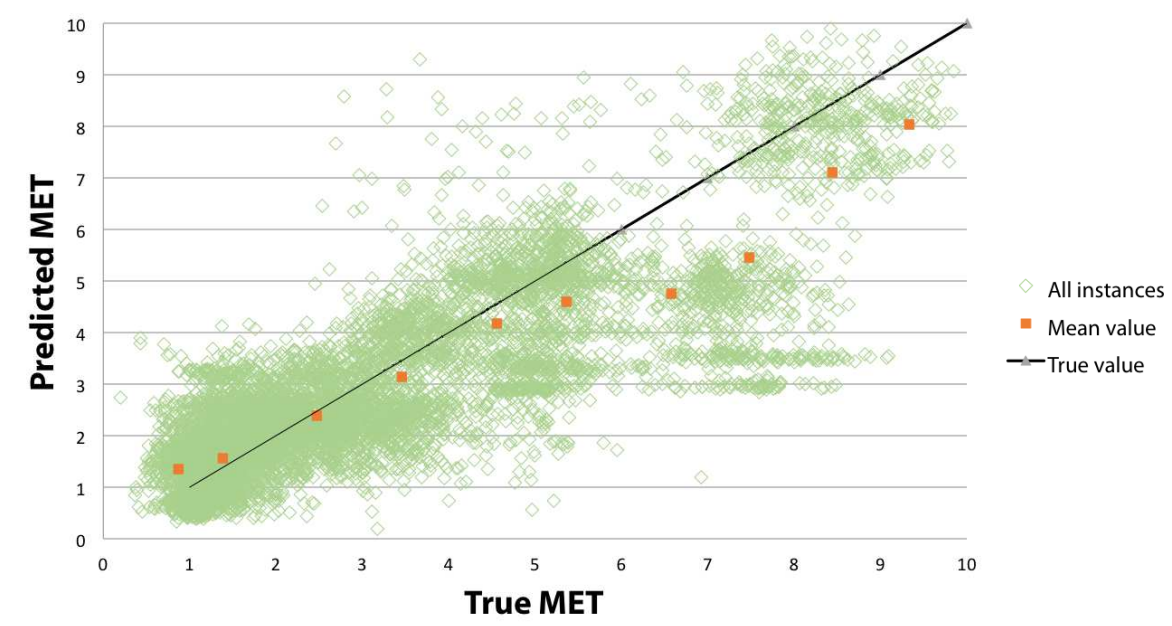

Fig. 6. Predicted MET vs. true MET for smart-phone configuration. 
(the activity recognition model did not perform with satisfactory results at this point). The future work can focus on activity recognition using smart phone and using it to improve EE estimation.

Figure 6 shows the estimation error for the smart-phone set model trained with SVR. We can observe that the error is low only for the activities with low and moderate intensity from 1.5 MET to 6 MET. The error occurs for the activities with high-moderate and high intensity. MAE is lower than SenseWear's, while MAPE is higher, but still comparable to SenseWear's.

Table 3. Results of machine-learning algorithms for regression for each configuration. The error is calculated as MAE. The compared algorithms are: support vector regression (SVR), linear regression (LR), multilayer perceptron (MLP), M5Rules, M5P, REPTree.

\begin{tabular}{lcccccc}
\hline & \multicolumn{5}{c}{ Algorithms } \\
\cline { 2 - 7 } Configuration & SVR & LR & MLP & M5Rules & M5P REPTree \\
\hline Full set & 0.65 & 0.66 & 0.70 & 0.69 & 0.68 & 0.68 \\
Embedded set & 0.76 & 0.78 & 0.83 & 0.84 & 0.83 & 0.81 \\
Smart phone set & 0.83 & 0.88 & 1.04 & 1.05 & 1.04 & 1.01
\end{tabular}

Table 4. Results of the final models for each set presented in MAE and MAPE.

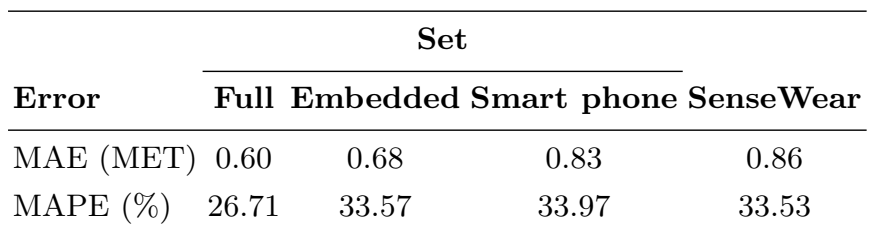

\section{Discussion and Conclusion}

The paper analysed the feasibility of EE estimation during free-living activities using machine-learning, while using dedicated and smartphone inertial sensors. The study gradually decreased the number of sensors used, and evaluated the error for each of the following configurations: (i) the full set with two Shimmer inertial sensors (attached to the chest and thigh) and the heart-rate sensor from the Zephyr chest-strap, (ii)the embedded set with the embedded inertial and heart-rate sensors from the Zephyr chest strap, and the embedded inertial sensor from a smart phone, and (iii) the smart-phone set with the embedded inertial sensor from a smart phone. Additionally, the energy expenditure estimation 
was compared to a commercial device SenseWear, which also estimates energy expenditure and is currently one of the most accurate devices for free-living activities.

As expected, the best results were obtained using the richest sensor configuration, that is, dedicated inertial sensors and a heart rate sensor. The MAE for this configuration was 0.60 MET, which is 0.26 MET lower than MAE achieved by the SenseWear, while MAPE was lower by approximately 5 percentage points.

The second sensor-set configuration, which consists of embedded sensors placed at the same location as the sensors in the first configuration, achieved the MAE 0.08 MET higher than the first configuration, while still being lower than the SenseWear's by 0.18 MET. The MAPE increased and is comparable to SenseWear's MAPE.

The last sensor set configuration consists of a smart phone only, carried in the pocket. This configuration achieved the highest MAE and MAPE, thus confirming our hypothesis that a reduced sensor set cannot achieve top performance. However, the MAE and MAPE are approximately 0.03 MET lower and 0.5 percentage point higher than Senswear's, respectively. This result leads to the conclusion that smart phone inertial sensors can estimate the energy expenditure roughly as reliably as the current state-of-the-art commercial devices, while using fewer sensors and without requiring a dedicated device.

We used essentially the same methodology with all three sensor set configurations, and we are confident that this methodology can be used with other device as well, not only with dedicated sensors and smart phones. Considering the proliferation of sensors in everyday devices, this observation is increasingly relevant. The future work, apart from the research on features for energy expenditure, will focus on normalizing the smart phone orientation, so that the energy expenditure estimation will be equally reliable regardless of the orientation. Our plans also include and additional effort on activity recognition using only a smart phone, for which a proper orientation normalisation is particularly important. As demonstrated in richer configuration sets, when activities can be recognised with relatively high accuracy, the energy expenditure estimation models achieve lower error rates.

\section{References}

1. World health Organization, HEPA, http://www.euro.who.int/

2. Cooper, S. B., Bandelow, S., Nute, M. L., Morris, J. G., Nevill, M. E.: The effects of a mid-morning bout of exercise on adolescents' cognitive function. Mental Health and Physical Activity, 5, 183-190 (2012)

3. Hamer, M., Stamatakis. E.: Objectively assessed physical activity, fitness and subjective wellbeing. Mental Health and Physical Activity, 3, 67-71 (2010)

4. Kohl, H.W., Craig, C.L., Lambert, E.V., Inoue, S., Alkandari, J.R., Leetongin, G., Kahlmeier, S.: The pandemic of physical inactivity: global action for public health. The Lancet, 380, 294-305 (2012)

5. Webb, P., Annis, J. F., Troutman Jr., S. J.: Energy balance in man measured by direct and indirect calorimetry. American Journal of Clinical Nutrition, 33, 12871298 (1980) 
6. Levine, J. A.: Measurement of Energy Expenditure. Public Health Nutrition, 8, 1123-1132 (2005)

7. Speakman. J.: Doubly labelled water: Theory and practice. Springer (1997)

8. Nintendo Wii, http://www.nintendo.com/wii

9. EA Sports Active 2, http://www.ea.com/ea-sports-active-2

10. Lustrek, M., Gjoreski, H., Kozina, S., Cvetkovic, B., Mirchevska, V., Gams, M.: Detecting Falls with Location Sensors and Accelerometers. In: Proceedings of the Twenty-Fifth AAAI Conference on Artificial Intelligence and the Twenty-Third Innovative Applications of Artificial Intelligence, pp. 1662-1667. AAAI press, (2011)

11. Kaluza, B., Cvetkovic, B., Dovgan, E., Gjoreski, H., Gams, M., Lustrek, M.: Multiagent Care System to Support Independent Living. International Journal on Artificial Intelligence Tools. Accepted for publication (2013)

12. Aminian, K., Mariani, B., Paraschiv-Ionescu, A., Hoskovec, C., Bula, C., Penders, J., Tacconi, C., Marcellini, F.: Foot worn inertial sensors for gait assessment and rehabilitation based on motorized shoes. In: Annual International Conference of the IEEE Engineering in Medicine and Biology Society, EMBC, pp.5820-5823, IEEE press, (2011)

13. Owusu, E., Han, J., Das, S., Perrig, A., Zhang, J.: ACCessory: password inference using accelerometers on smart phones. In: Proceedings of the Twelfth Workshop on Mobile Computing Systems and Applications, pp. 91-96. ACM, New York, (2012)

14. Cosmed http://www. cosmed.com/

15. SenseWear, http://sensewear . bodymedia.com/

16. Andre D., Wolf, D.L.: Recent Advances in Free-Living Physical Activity Monitoring: A Review. J. Diabetes Sci. Technol. 1(5), 760-767 (2007)

17. ACCUPEDO, http://play.google.com/store/apps/details?id=com.corusen. accupedo.te

18. Leijdekkers, P., Mark V.G.: User Adoption of Mobile Apps for Chronic Disease Management: A Case Study Based on myFitnessCompanion, In: Donnelly, M., Paggetti, C., Nugent, C., Mokhtari, M. (eds.) Impact Analysis of Solutions for Chronic Disease Prevention and Management. LNCS, vol. 7251, pp. 42-49. Springer, Heidelberg (2012)

19. Ainsworth, B. E., Haskell, W. L., Whitt, M. C., Irwin, M. L., Swartz, A. M. et al.. Compendium of physical activities: An update of activity codes and MET intensities, Medicine and Science in Sports and Exercise, 32, 498-516 (2000)

20. Heil, D. P.: Predicting activity energy expenditure using the actical activity monitor. Research quarterly for exercise and sport, 77, 64-80 (2006)

21. Bouten, C. V., Westerterp, K. R., Verduin, M., Janssen. J. D.: Assessment of energy expenditure for physical activi (1994)

22. Crouter, S. E., Clowers, K. G., Bassett, D. R.: A novel method for using accelerometer data to predict energy expenditure. Journal of applied physiology, 100, 13241331 (2006)

23. Actigraph, http://www. actigraphcorp.com/

24. Nike+, http://nikeplus.nike.com/plus/

25. Shimmer research, http://www.shimmer-research.com/

26. Zephyr Biohraness, http://www.zephyranywhere.com/products/bioharness-3/

27. Samsung Galaxy SII http://www.samsung.com/

28. Kozina, S., Gjoreski, H., Gams, M., Lustrek, M.: Three-layer Activity Recognition Combining Domain Knowledge and Meta-classification. J. Med. Biol. Eng., available online (2013) 
29. Cvetkovic, B., Kaluza, B., Lustrek, M., Gams, M.: Multi-Classifier Adaptive Training: Specialising an Activity Recognition Classifier Using Semi-supervised Learning. In: Patern, F., Ruyter, B., Markopoulos, P., Santoro, C., Loenen, E., Luyten, K. (eds.) Ambient Intelligence 2012. LNCS, vol. 7683, pp. 193-207. Springer, Heidelberg (2012)

30. Tapia, E. M.: Using machine learning for real-time activity recognition and estimation of energy expenditure. Ph.D. Thesis, Massachusetts Institute of Technology (2008)

31. Robnik-Sikonja, M., Kononenko, I.: Theoretical and empirical analysis of ReliefF and RReliefF. Machine Learning, 53, 23-69 (2003)

32. Hall, M., Frank, E., Holmes, G., Pfahringer, B., Reutemann, P., Witten, I. H.: The WEKA Data Mining Software: An Update. SIGKDD Explorations, 11, 10-18 (2009)

33. Lustrek, M. and Cvetkovic, B. and Kozina, S.: Energy expenditure estimation with wearable accelerometers. In: 2012 IEEE International Symposium on Circuits and Systems (ISCAS), pp. 5-8. IEEE press, (2012) 